\title{
Epidermolyses bulleuses et anomalies moléculaires de la kératine
}

Sous le nom d'épidermolysc bulleuse simplex on rasscmble un groupe de maladies cutanées dont le caractère commun est la formation de bulles qui tendent ensuite à se décoller. Les formes familiales sont en règle à transmission autosomique dominante. Lc décollement cutané est parfois spontané, mais le plus souvent est la conséquence d'un traumatisme local. On en distingue habituellement trois formes : deux d'entre elles sont d'apparition néonatale et sont généralisées; la plus grave, dite de Dowling-Meara, dont les bulles prennent un caractère herpétiforme, et celle de Koebner ; la troisième forme, ditc de Weber-Cockayne, est plus bénigne, apparaît plus tardivement et reste localisée aux paumes des mains ct aux plantes des pieds. Un ensemble de travaux a fait évoquer l'hypothèse d'anomalics de la kératinc à l'origine de ccrtains types de la maladie.

Les kératines sont des protéines très hétérogènes, codécs par au moins une vingtaine de gènes et comportant en outre des pscudogènes. Ellcs possèdent toutes un domaine central en hélice $\alpha$ de 310 acides aminés, flanqué d'extrémités $\mathrm{C}$ - et $\mathrm{N}$-terminales variables. Elles se rangent on deux types - I et II -, génétiquement et chimiquement différents. La séquence de leur domaine central differe. Le type I est codé par des gènes rattachés au chromosome 17 en 17q 12-21; le type II, par des gènes portés par le chromosome 12 en 12q 11-13 [1]. Dans les filaments intermédiaires de la peau, les kératines sont organisées par paires avec une molécule de chaque type I ct II. Dans les cellules basales, on trouve la paire K14 (type I)-K5 (type II) que remplacent progressivement d'autres lules cutanées. L'ADNc de certaines kératines est connu depuis 1982 [2]. Unc brève récente $\left(\mathrm{m} / \mathrm{s} n^{\circ} 4\right.$, vol. 7 , p. 390) rapportait des expériences de transgenèse de Vassar et al. (Chicago, IL, USA), montrant que l'introduction de gènes tronqués de kératine provoquait chez les souris receveuses des lésions cutanées rappelant celles de l'épidermolyse bulleuse. L'autcur de cette brève prévoyait donc une recherche d'anomalies de la kératine dans cette maladic. C'est chose faite aujourd'hui, grâce aux travaux de ce même groupe d'Elaine Fuchs. Coulombe et al. [3] ont examiné six sujets, dont trois classés comme forme de Dowling-Meara et trois comme forme de Kocbner sur la base de données cliniques et ultrastructurales. Les cellules en culture des trois premiers présentaient des filaments nettement plus courts que les témoins. A partir de kératinocytes en culture de deux de ces sujets, on prépara le messager et on amplifia l'ADNc dérivé de cet ARNm; le domaine en hélice $\alpha$ fut ensuite analysé. Dans les dcux cas, on trouva unc mutation portant sur l'acide aminé 125 , situé près de l'extrémité $\mathrm{N}$-terminale du domaine en hélice $\alpha$. Chez le premier malade, c'était le

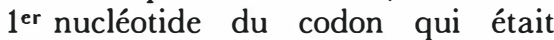
muté (nt $\mathrm{n}^{\circ} 433, \quad \mathrm{C} \rightarrow \mathrm{T}$, Arg $\rightarrow$ Cys); chez le sccond, c'était lc $2^{\mathrm{c}}$ nucléotide (nt $\mathrm{n}^{\circ} 434, \mathrm{G} \rightarrow \mathrm{A}$, Arg $\rightarrow$ His)

Les vérifications habituelles montrèrent l'absence de ccs mutations chez les témoins, mais révélèrent la fréquence d'un polymorphisme $\mathrm{C} \leftrightarrow \mathrm{T}$ au nt 429 , siégeant à la $3^{\mathrm{e}}$ base du codon 123 et laissant inchangé l'acide aminé Asn ; les autcurs sont cependant frappés du nombre de mutations dans cette région ct soulèvent l'hypothèse d'un point chaud de mutations.
La présence d'une mutation ponctuclle ne démontrant pas son rôle dans la genèse de la maladie, les auteurs ont cherché à renforcer leurs arguments. Ils font d'abord remarquer la pérennité de l'Arg 125 de l'escargot à l'homme ; mais la confirmation décisive fut obtenue en provoquant la mutation 125 Arg-Cys dans un ADNc de kératine normale, marquée par de la substance $\mathrm{P}$, et en l'utilisant pour transfecter des kératinocytes en culture, révélée par des anticorps appropriés. Alors que le témoin P-K14 n'a aucun effet nocif, le mutant détruit le réseau de kératine, provoquant l'apparition de lésions semblables à celles des malades.

Il semble donc que des mutations survenant dans le domaine en hélice $\alpha$ d'une kératine soient capables de provoquer des maladies cutanées. Ce domaine contient des répétitions d'un heptapeptide dont les positions 1 et 4 sont occupées par des résidus hydrophobes. L'Arg 125 est en position 7 d'un de ces heptapeptides, position qui pourrait être critique pour l'assemblage des filaments. Il est évident que cette recherche ne fait que commencer, et que d'autres anomalies des gènes de $\mathrm{K} 5$ et $\mathrm{K} 14$, et probablement d'autres gènes, seront bientôt mises en évidence. Déjà, par des études de liaison génétique, le défaut dans une famille atteinte de la forme de Koebner s'est montré résider sur le chromosome 17 dans une région qui englobe le gène de la kératine $\mathrm{K} 14$, et dans une famille du type Weber-Cockayne sur le chromosome 12 dans une région qui porte le gène de la kératine K5 [4]. Il est donc vraisemblable que ces travaux sont destinés à prendre une extension considérable.

Il ne faut cependant pas exclure 
d'autres origines génétiques pour les épidermolyses bulleuses. C'est ainsi que dans une famille d'origine irlandaise [5] présentant une forme de Koebner, une liaison génétique a été trouvéc avec le bras long du chromosome 1 (lod score ne dépassant toutefois pas 3). Par aillcurs, d'autres formes d'épidermolyse bulleuse pourraient avoir un mécanisme très différent. Un exemple tout récent en est fourni par Ryynänen et al. (Philadelphic, PA, USA), qui ont étudié une forme dystrophique avec formation de cicatrices; dans une famille finlandaise, une liaison génétique étroite a été établic avec le gène du collagène type VII (COL VII $A 1$ ), dont les auteurs [6] montrent la localisation sur le chromosome 3.

J.-C. D.

Note : tout récemment, dans une famille finlandaise, une lésion a été trouvée avec le chromosome $12|7|$
1. Frézal J, Baule MS, de Fougerolle T. Genat las. Paris : Inserm/John Libbey Eurotext, 1991: 623-719.

2. Hanukoglu I, Fuchs E. The cDNA sequence of a human epidermal keratin : divergence of sequence but conservation of structure among intermediate filament proteins. Cell $1982 ; 31$ : 243-52.

3. Coulombe PA, Hutton ME, Letai A Hebert A, Paller AS, Fuchs E. Point mutations in human keratin 14 genes of epidermolysis bullosa simplex patients : genetic and functional analysis. Cell 1991; 66: 1301-11

4. Bonifas J, Rothman AL, Epstein E. Linkage of epidermolysis bullosa simplex to probes in the region of keratin gene clusters on chromosomes 12q and 17q. I Invest Dermatol 1991; 96 : $550 \mathrm{a}$

5. Humphries MM, Shiels D, Lawler M, et al. Autosomal dominant simplex epidermolysis bullosa : evidence for linkage to genetic markers on chromosome 1. Genomics 1990; 7: 377-81.

6. Ryynänen M, Knowlton RG, Parente MG Chung LC, Chu ML, Uitto J. Human type VII collagen : genetic linkage of the gene COL VII AI on chromosome 3 to dominan dystrophic epidermolysis bullosa. Am J Hum Genet $1991 ; 49$ : 797-803.

7. Ryynänen M, Knowlton RG, Witto J, Mapping of epidermolysis bullosa simplex to chromosome 12. Am J Hum Genet 1991 ; 49 978-84.

$\mathrm{m} / \mathrm{s} n^{\circ} 10$, vol. 7 , décembre 91

\section{BRÈVES}

Mutations dans le promoteur du gène $R b$ dans des cas de rétinoblastomes familiaux. Les formes familiales du rétinoblastome sont dues à la transmission, à l'état hétérozygote, d'une mutation du gène de prédisposition au rétinoblastome (gène $R b)\left([1]\right.$ et $\mathrm{m} / \mathrm{s} n^{\circ} 8$, vol. 4 , p. $520 ; n^{\circ} 4$, vol. 5 , p. 260). L'allèle normal est soit perdu, soit altéré au niveau des tumeurs. La majorité des mutations du gène $R b$ sont des délétions ou des modifications de la structure protéique. Une équipe américaine de Boston (MA) et Pittsburgh (PA) a étudié la région du promoteur chez 111 malades chez lesquels aucune altération grossière du gène $R b$ n'avait été mise en évidence. Dans deux formes familiales, des mutations étaient trouvées, une transversion $G \rightarrow T 189$ bases avant le site d'initiation de la transcription et une transition $\mathrm{G} \rightarrow$ A 9 bases plus en amont. Dans ces deux familles, la pénétrance de cette mutation était incomplète, ce qui signifie que tous les porteurs ne développaient pas de rétinoblastome [2]. Les deux mutations intéressent des éléments d'ADN sur lesquels se fixent des facteurs de transcription, ATF/CREB et Sp1. ATF/CREB correspond à une grande famille de protéines reconnaissant, notamment, les éléments de réponse à l'AMP cyclique (CRE) et des éléments relayant l'effet transactivateur de la protéine adénovirale E1A sur certains promoteurs alors que $\mathrm{Sp} 1$ se fixe à des éléments d'ADN riches en GC et présents au niveau de très nombreux promoteurs, notamment ceux des gènes ubiquitaires. Ces deux mutations annulent pratiquement la fixation d'ATF/CREB et de Sp1 à leurs cibles dans le promoteur du gène $R b$. Les auteurs ont pu ensuite démontrer que ces deux mutations avaient, comme on pouvait s'y attendre, d'importantes conséquences fonctionnelles sur l'activité du promoteur qui était réduite à 6-7 \% de la normale par l'une ou l'autre [2]. Les auteurs expliquent la faible pénétrance de ces mutations du promoteur par le fait qu'elles n'entraînent pas une inactivité totale de l'allèle muté. De plus, le mécanisme expliquant le développement des tumeurs dans ces formes constitutionnelles de rétinoblastome est l'inactivation de l'allèle normal, souvent par remplacement de tout ou partie du chromosome normal par son équivalent du chromosome muté. Dans ce cas, la faible activité résiduelle de deux allèles portant une identique mutation promotrice pourrait être suffisante pour éviter la prolifération incontrôlée.

[1. Junien C. médecine/sciences 1986 ; 2 : 238-45.]

[2. Sakai T, et al. Nature $1991 ; 353$ : 83-6.]

-1]ne myxomatose contraceptive contre les lapins australiens. La prolifération des lapins est devenue un fléau considérable en Australie. Les ravages provoqués par cet animal prolifique ont justifié l'introduction, en 1950, du virus de la myxomatose en Australie [1]. Cette épizootie provoqua une diminution très importante du nombre de lapins, mais ces animaux sont à présent devenus résistants à la myxomatose et leur nombre augmente à nouveau. Le CSIRO, l'organisation australienne nationale de recherche, envisage maintenant d'utiliser le virus de la myxomatose pour véhiculer un gène codant pour une protéine de la tête des spermatozoïdes indispensable à la fécondation des ovocytes. Les femelles développeraient alors des anticorps et ne pourraient donc être fécondées par les spermatozoïdes des mâles. Le but est alors de provoquer une épizootie de stérilité qui semble la mieux à même de stopper la réaugmentation du nombre de lapins australiens.

[1. Thwaites T. New Scientist 1991; 132 : 18.]
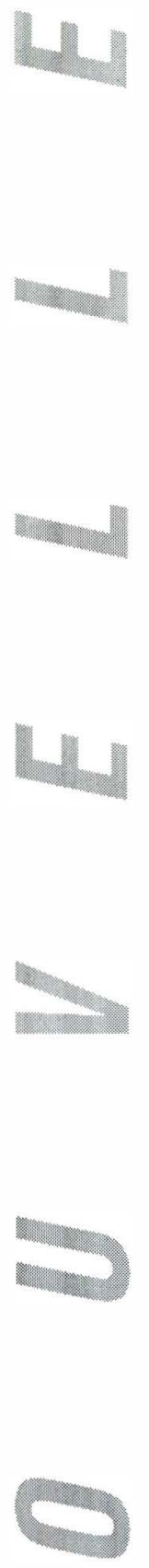

1097 